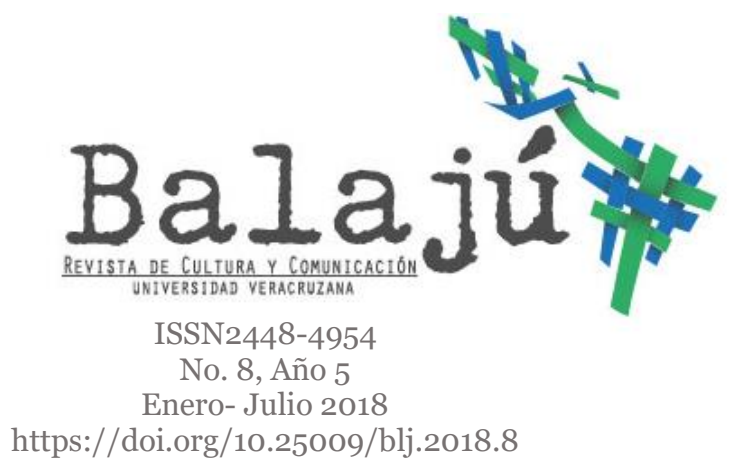

\title{
El debate público sobre el conocimiento y la información dentro de Wikipedia en español: Apuntes sobre la participación de los editores y voluntarios de Wikimedia México
}

The public debate about knowledge and information on the Spanish Wikipedia: Notes on the participation of Wikimedia Mexico's editors and volunteers

\author{
Luis Álvarez Azcárraga 1 \\ https://orcid.org/oooo-0002-7362-985X
}

\begin{abstract}
RESUMEN: Wikipedia en español es una enciclopedia participativa en la que muchas agendas informativas y opiniones se enfrentan para decidir los contenidos. Este proceso se complica en un entorno abierto en el que hay actores diversos, desde usuarios comprometidos con el proyecto, empresas de relaciones públicas, políticos, empresas y vándalos. En este artículo se mostrará una visión de esta problemática desde la perspectiva de los usuarios y voluntarios de Wikimedia México, quienes han encontrado estrategias de participación política, desde mecanismos de votación hasta herramientas para expulsar a usuarios problemáticos.
\end{abstract}

PALABRAS CLAVE: Wikipedia; participación; conocimiento libre; cultura colaborativa; deliberación

ABSTRACT: Spanish Wikipedia is a participatory encyclopedia in which many agendas
and opinions are faced to decide the content; however, this process is complicated in an
open environment with diverse actors, from committed users, PR companies, politicians,
and vandals. This article will show a vision of this problem from the perspective of the
users and volunteers of Wikimedia Mexico, who have found strategies of political
participation, from voting mechanisms to tools to expel problematic users. KEYWORDS: Wikipedia; participation; free knowledge; collaborative culture; deliberation

\footnotetext{
${ }^{1}$ Universidad Autónoma de Aguascalientes, México. Correo electrónico: luisalvaz@ gmail.com
} Fecha de recepción: 04/08/2017. Aceptado: 26/03/2018 


\title{
El debate público sobre el conocimiento y la información dentro de Wikipedia en español: Apuntes sobre la participación de los editores y voluntarios de Wikimedia México
}

\author{
No podemos pelear contra el encarcelamiento \\ sin memoria ni imaginación. \\ Bassel Khartabil (1981-2015)
}

\section{Introducción}

El presente artículo expondrá, a través de la visión de los editores y voluntarios de Wikimedia México, parte de la construcción del debate público dentro de Wikipedia en español, desde la creación de artículos, así como las discusiones que se generan por los desacuerdos en torno al contenido.

El proceso de apropiación del debate público no ocurre de forma uniforme, en ciertos espacios sociales la apropiación es escasa o nula, debido al control de la información o a su manipulación por parte de ciertos actores; en otros espacios, como en las plataformas sociodigitales de la web, la apropiación puede ser tan abierta que resulta caótica, sin estructura y con el temor constante de la repetición de notas informativas falsas o de escasa relevancia.

Aunque en la práctica las comunidades virtuales funcionan de manera interconectada, resulta conveniente estudiarlas de manera independiente, para identificar sus cualidades inherentes, así como la forma en que los usuarios emplean la información, construyéndola y poniéndola en circulación, encontrando sus estrategias, reglas y problemáticas particulares.

Wikipedia, al ser una enciclopedia abierta, no es la excepción; al ser un proyecto colaborativo en el que cualquier persona puede contribuir anónimamente, o con un avatar, ha creado una organización y estructura que algunos llaman meritocrática (Ortega Soto y Rodríguez López, 2011) y otros adhocrática (Bruns, 2008). Esto quiere decir que dentro del sitio, las relaciones no son jerárquicas, aunque tampoco es totalmente horizontal y abierta, pues existe una reglamentación interna en la que, aunque algunos usuarios no la conozcan, hay otros que se encargan de que se cumpla.

El objetivo de este artículo es mostrar analíticamente las herramientas de deliberación de algunos de los usuarios de Wikipedia en español, así como de los voluntarios de Wikimedia México, lo que no sólo incentiva la participación de nuevos usuarios dentro de la enciclopedia, sino que también la han convertido en un instrumento para el activismo por el conocimiento libre y la inclusión de otros conocimientos que han sido excluidos en los campos académicos tradicionales.

\section{Metodología}

El presente artículo parte de una investigación etnográfica que tomó como informantes a usuarios de Wikipedia en español y a voluntarios de Wikimedia México. Como herramientas metodológicas (Tabla 1) se recurrió a un estudio de caso (Stake, 1998) para analizar profundamente el comportamiento de los voluntarios y usuarios en el ámbito presencial dentro de eventos de edición (editatones) y su participación dentro de la Wikipedia en español. Para esto, se 
realizó una etnografía de Internet (Hine, 2015), a través de la variante de la etnografías de la participación (Cefaï, et al., 2012). La etnografía de la participación permite rastrear las interacciones de los usuarios y voluntarios involucrados en su propio terreno a través de la observación participante. "Este encarnada y contextualizada de la experiencia es tanto más valiosa que el acceso mediante la observación directa a una dimensión situaciones sociales que no son percibidas como políticas, ni por quienes participan, ni siquiera, a menudo, por quienes les observan" (Cefaï, et al., 2012: 11)

Tabla 1. Herramientas metodológicas e informantes de donde se obtuvieron los datos de esta investigación

\begin{tabular}{|c|c|c|}
\hline $\begin{array}{c}\text { Herramienta } \\
\text { metodológica }\end{array}$ & Entorno & Información a analizar \\
\hline \multirow{2}{*}{$\begin{array}{c}\text { Etnografía virtual y } \\
\text { observación participante } \\
\text { en Wikipedia en español }\end{array}$} & $\begin{array}{c}\text { Artículos editados por } \\
\text { los informantes } \\
\text { (voluntarios de } \\
\text { Wikimedia México) }\end{array}$ & Número de artículos \\
\cline { 2 - 3 } & $\begin{array}{c}\text { Interacción entre } \\
\text { usuarios y discusiones }\end{array}$ & Tipo de interacción \\
\cline { 2 - 3 } & $\begin{array}{c}\text { Votaciones internas / } \\
\text { Deliberaciones }\end{array}$ & Decisiones tomadas \\
\hline \multirow{2}{*}{$\begin{array}{c}\text { Observación participante } \\
\text { y entrevistas en } \\
\text { profundidad }\end{array}$} & $\begin{array}{c}\text { Encuentros de edición } \\
\text { (editatones) }\end{array}$ & $\begin{array}{c}\text { Número de artículos / } \\
\text { artículos borrados / } \\
\text { interacciones }\end{array}$ \\
\cline { 2 - 3 } & Talleres de edición & Interacción \\
\hline
\end{tabular}

Además de las interacciones de los usuarios en Wikipedia, se analizaron sus contribuciones, a partir de las categorías de análisis: participación políticomediática, conocimiento libre y cultura de la colaboración. Esta información ayudó a encontrar tanto las asimetrías en la participación, como las pugnas internas por el contenido y cómo se organizan y debaten los editores para construir un discurso de la información relevante que siempre es susceptible a ser modificada.

\section{Distribución abierta y cerrada del poder en red}

Si definimos al poder como lo hace Castells (2012), como el proceso de comunicación que crea significados en la mente del ser humano a través de las relaciones o redes socioculturales, ya sea en el plano de la comunicación global, local, de masas y en lo que se denomina 'autocomunicación de masas'; esto quiere decir que el poder ocurre en diversos planos, de manera compleja y, siempre, a través del discurso y el intercambio simbólico.

El poder, entonces, es relacional, y dependiendo del nivel de participación permitido en un sistema de interacciones sociales, el poder será distribuido y compartido por más o menos actores. En una sociedad cerrada, tomando en cuenta la concepción de Popper (1992), el poder se encuentra en pocas manos, mientras en un sistema abierto la distribución se comparte entre más miembros de la comunidad. Sin embargo, como lo señalan Mouffe (2005) y Rancière (2015), las democracias representativas simulan una distribución equitativa del poder, 
dejando la toma de decisiones a unos pocos individuos. Una participación democrática que permita una toma de decisiones distribuida a gran parte de la población, como señala Dahlgren (2013), no está garantizada por las democracias actuales, y dependen más que de las relaciones de poder tradicionales, así como de las redes ciudadanas que emergen de manera coyuntural en la red.

Las relaciones en red, pues, requieren de interacciones complejas en las que el poder se redistribuye; sin embargo, hay nodos que concentran un mayor número de interacciones y otros que se encuentran aislados. Esta capacidad de concentrar la información y de incidir en el resto de la red, es el poder de la comunicación en red, y se puede encontrar en al menos cuatro formas (Castells, 2012: 72)

- Poder de conectar en red. Inclusión o exclusión de los actores dentro de la red.

- Poder de la red. Reglas y protocolos que hay dentro de la red, que casi siempre benefician a pocos actores o nodos del sistema.

- Poder en red. Se refiere a cómo opera el poder en diversos niveles de la red, pues aunque se concentra más en ciertos nodos, se distribuye en el sistema en diversos niveles.

- Poder para crear redes. Dividido en los 'programadores', quienes construyen las redes; y los 'enlaces', que pueden conectar a diversos actores dentro de la red.

Ningún sistema, sin embargo, opera de forma autónoma. Las redes pueden estudiarse de manera aislada, pero siempre tomando en cuenta su relación con redes más amplias. Dentro de Wikipedia, por ejemplo, es posible estudiar (como es el caso de este estudio) un grupo de usuarios y voluntarios que, sin embargo, dependen de una infraestructura más amplia que, a su vez, también está interconectada a muchos otros sistemas de intercambio de información. Los usuarios que trabajan de manera colectiva y, hasta cierto punto, horizontal (creando una suerte de democracia participativa); se enfrentan a las relaciones con la Fundación Wikimedia, de quienes dependen para que la infraestructura funcione las 24 horas del día. Pero también dependen de un sistema de protocolos de la red, de motores de búsqueda y de los servicios de las compañías proveedoras de Internet, así como de los sistemas tradicionales de intercambio y validación del conocimiento. Todos estos intercambios socio-digitales influyen en las decisiones de los usuarios; sin embargo, dichas decisiones también influirán en el comportamiento de los lectores, de la información que se comparte en las redes sociales, en los sistemas educativos y en las formas en las que las personas construyen su opinión sobre temas de interés público.

\section{Autoría tradicional vs. Inteligencia colectiva}

La inteligencia colectiva, enunciada por Lévy (2004) y seguida por Jenkins (2009), hace alusión a una relación social en la que la creación colectiva puede ser más valiosa y efectiva que la creación individual, o incluso con la idea de que lo que hasta entonces se había considerado como creación individual en realidad siempre es parte de un sistema de interacciones e interrelaciones, en ocasiones no reconocidas. 
Sin embargo, a diferencia de otro tipo de formas de crear y de relacionarse, la de la inteligencia colectiva requiere que haya un sistema horizontal de interacciones, a diferencia del sistema autoral en el que el 'creador' se encuentra en un escaño más alto que sus lectores en la jerarquía creativa. Rancière señala que la única forma de que exista la igualdad es reconocer en primera instancia la igualdad de las inteligencias', que “...es el vínculo común del género humano, la condición necesaria y suficiente para que una sociedad de hombres exista" (Rancière, 2003: 42). Esta posible igualdad de las capacidades no quiere decir que todos tengan las misma capacidad "...sino que hay una distribución diferenciada de las formas de implicancia de una inteligencia que es la misma para todo el mundo" (Rancière, 2014: 163). En otro texto, Rancière (2010) reflexiona sobre la posibilidad de que el 'espectador' se emancipe del sistema tradicional, pero para esto se requiere que el autor realice una obra lo suficientemente abierta para que exista una participación activa; algo similar a la propuesta de Augusto Boal con su 'espec-actor' con el teatro del oprimido (Boal, 2013).

La 'inteligencia colectiva' en la red, más allá de ser un fenómeno de colaboración armónica, describe un fenómeno de democracia deliberativa o de antagonismo, en palabras de Mouffe; es decir, de espacios de apropiación de un canon para transformarlo o adaptarlo a las necesidades de un grupo de usuarios. Esto se relaciona con lo que Jenkins (2006) denomina cultura participativa, pues en la cultura popular o la tradición oral un grupo de personas transforma los contenidos, en ocasiones contraviniendo o transgrediendo las reglas canónicas de un sector académico. La cultura participativa es definida en oposición a otras formas de cultura letrada, las cuales:

(...) limitan el acceso a los medios de producción cultural y de circulación, que fragmentan y aíslan al público en vez de proveerlos oportunidades de crear y compartir cultura, y que construyen jerarquías que hacen difícil para muchos ejercer cualquier influencia significativa sobre las decisiones fundamentales que afectan sus vidas. (Jenkins, Ito y boyd, 2016: 194)

En este sentido, la cultura participativa dependería tanto de lo que Carpentier (2011) considera como ligeras desigualdades dentro de relaciones de horizontalidad, como del compromiso del grupo por contrarrestar otras formas de dominación estructural que ejerce las instituciones gubernamentales, los medios comerciales y otras organizaciones jerárquicas como los círculos académicos.

En el caso de Wikipedia, se ha estudiado este fenómeno desde diversas perspectivas, como el caso de Jemielniak (2014) quien a través de una etnografía en Wikipedia en inglés y polaco analizó la manera en que los editores se organizan, crean alianzas, tienen conflictos y deliberan; o el estudio de Lih (2009), con el que se da cuenta de cómo ha crecido Wikipedia en diferentes versiones, y algunos de los principales conflictos dentro de la comunidad, como el de Wikipedia en español cuando se propuso que hubiera publicidad en el sitio, provocando que muchos usuarios se retiraran y crearan una wiki alternativa: la Enciclopedia Libre Universal en Español, o también la forma en que los usuarios se organizaron para protestar contra una iniciativa de ley que ponía en riesgo el proyecto, SOPA (Stop Online Piracy Act). 
O'Neil (2009) ve en Wikipedia un lugar donde nuevas formas de centralizar el control del contenido emergen de manera alternativa, a través de liderazgos y alianzas entre usuarios, algo similar a lo que señalan Ortega Soto y Rodríguez López (2011) que ubican a usuarios más comprometidos que otros en la creación del contenido de Wikipedia en español, quienes reciben incentivos simbólicos, así como prestigio dentro del proyecto que los impulsa a continuar mejorando el contenido de la enciclopedia.

Cabe destacar, que en un principio se pensó en Wikipedia como una herramienta destinada a crear artículos que fueran corregidos por expertos en las áreas temáticas de la enciclopedia; sin embargo, esta idea fracasó "debido a la lentitud de los académicos invitados a participar como editores." (Van Dijck, 2016: 222-23). Wikipedia se convirtió así, en un modelo en el que cualquier editor pudiera participar, por lo que los fundadores, Jimmy Wales y Larry Sanger tuvieron que crear un protocolo para que el modelo no se saliera de control, como la aparición de los 'cinco pilares', que entre otras cosas, señalaban que el contenido plasmado en Wikipedia debía tener relevancia enciclopédica y estar escrito desde un punto de vista neutral (rasgos distintivos del enciclopedismo tradicional y la academia).

Desde este punto de vista, aunque Wikipedia proponga la libertad como elemento fundacional, y la posibilidad de que cualquier persona pueda editarla, también posee elementos que enfrentan a la autoría tradicional con un modelo colaborativo en el que el anonimato y la contribución voluntaria por un bien común son elementos fundamentales del proyecto.

\section{Conocimiento libre}

Free as in freedom / free as in speech

La libertad de compartir el software radica en que el código no sólo está abierto, sino que se puede reutilizar, ya sea para mejorarlo o para crear nuevas versiones del mismo (Stallman, 2010). Esto quiere decir que el carácter de 'libre' no está en la gratuidad (que puede existir o no), sino en que no posee restricciones definidas por el mercado o por un sector gubernamental, sino por la comunidad.

Esta misma capacidad se aplica usualmente para el concepto de cultura libre, contenido libre y conocimiento libre, conceptos que se tratarán a través de diversos autores y concepciones (tanto legales como teóricas).

Se han intentado definir al conocimiento y cultura libre desde una posición pragmática, que tenga siempre como horizonte el marco legal estadounidense, o al menos los marcos legales esenciales de los países capitalistas (Lessig, 2005). Es decir, alrededor del concepto de cultura libre subyace el hecho de que los contenidos y procesos culturales se han convertido en mercancía y, por ende, tienen un dueño que no necesariamente es el autor, sino quien posee la licencia para explotar comercialmente dicho producto. En este sentido, y para los críticos del modelo mercantil, el conocimiento ha dejado de ser un bien común para convertirse en mercancía, lo cual también habían denunciado algunos teóricos marxistas, como Adorno y Horkheimer (2007) con el concepto de industria cultural; o también Debord (2002) con la sociedad del espectáculo.

El concepto de libertad, sin embargo, supone varios retos conceptuales porque se trata de una categoría presente en muchos debates teóricos y filosóficos, 
que se acentuaron con las teorías políticas del liberalismo económico (Friedman, 2009; Smith, 1994) y la sociedad abierta (Popper, 1992). Sin embargo, el debate sobre la libertad se puede rastrear desde las discusiones teológicas del libre albedrío y sobre la ética en el ser humano. Al margen de estas problemáticas, algunos de los primeros autores interesados en definir la libertad dentro del marco de la producción de contenidos y, en específico, de productos digitales en el entorno virtual de Internet, propusieron las características que debería tener un producto cultural dentro del marco de los derechos digitales para que pudiera ser reutilizado con total libertad para así favorecer la reproducción del conocimiento, la información y los contenidos digitales. En este sentido surgen cuatro características esenciales (Freedom definition, 2015):

- La libertad de usar la obra y disfrutar de los beneficios de su uso.

- La libertad de estudiar la obra y de aplicar los conocimientos resultantes de ello.

- La libertad de hacer y redistribuir copias, de la obra completa o de parte de ella.

- La libertad de hacer cambios y mejoras, y de distribuir obras derivadas.

Estas características son importantes para la cultura libre en su praxis, pues a través de ellas se pueden generar normativas que funcionen en paralelo de la leyes de derechos de autor y del derecho a la copia (copyright - derechos de autor) vigentes en casi todos los países, para productos culturales, especialmente los del ámbito digital, y que puedan ser compartidos sin las limitantes legales tradicionales.

Sin embargo, para los términos de esta investigación, la cultura libre y el conocimiento libre, no son sólo categorías para compartir contenidos y recursos sin restricciones, sino que se trata de conceptos que aunque hayan surgido recientemente, hacen alusión a la capacidad inherente del conocimiento humano de replicarse tal como la teoría del meme (Dawkins, 1994) ha enunciado. Es decir, el conocimiento humano puede ser replicado, transformado y reutilizado de muchas formas, y solamente cuando dichos saberes se convierten en un formato físico (papel, registro fonográfico, obras de arte) devinieron en mercancía que podía ser controlada y cercada por unos cuantos (Attali, 1995; Shiner, 2004).

Como lo señala la organización Hipatia (s/f), el derecho al conocimiento libre es una propuesta que se encuentra en el artículo 27 la Declaración Universal de los Derechos Humanos de la Organización de las Naciones Unidas: "Toda persona tiene derecho a tomar parte libremente en la vida cultural de la comunidad, a gozar de las artes y a participar en el progreso científico y en los beneficios que de él resulten." ${ }^{2}$

\section{Conocimiento libre más allá del marco legal o 'cultura libre' como filosofía}

La concepción de conocimiento que se maneja dentro de la comunidad de Wikipedia no es una sola; en este sentido, lo que se denomina conocimiento libre,

\footnotetext{
2 Texto disponible en:

https://es.wikisource.org/wiki/Declaración_Universal_de_los_Derechos_Humanos 
no es solamente el hecho de generar contenido enciclopédico que sea capaz de ser compartido por cualquier persona bajo cualquier circunstancia, sino de que dichos contenidos sean susceptibles de ser confrontados, modificados y que incluso puedan criticarse por su falta de relevancia o porque son inexactos desde algún punto de vista. Esto quiere decir que el 'conocimiento libre' puede ser conocimiento científico pero también saberes de la cultura popular o contenidos que usualmente no son acreditados por las instituciones educativas, quienes por lo regular discriminan el conocimiento entre aquel que está acreditado y los 'saberes subordinados', los cuales no son parte del canon científico (Foucault, 1990).

La naturaleza del conocimiento científico (Bollier, 2008: 254) es más estructurada que la sabiduría de las multitudes, y al mismo tiempo tiene un orden jerárquico en el que la figura del autor es protagonista. En este sentido, mientras es primordial conocer quién escribió un artículo o paper para poder rastrear el reconocimiento que un grupo de especialistas le da a un nombre, así como a la institución de la cual procede dicha investigación así como las afirmaciones de la misma, un producto de la 'multitud inteligente' o del saber popular no requiere necesariamente que un autor se le reconozca, pues se trata, en principio de un autor colectivo.

A diferencia del concepto de conocimiento desde la tradición epistemológica que subraya la capacidad progresiva del saber humano, el conocimiento libre es un concepto de resistencia, que armoniza con el de cultura participativa, democracia deliberativa, ética hacker y el disenso. Esto quiere decir que es un concepto en construcción y con carácter aspiracional, que en la práctica funciona de manera paradójica, y que incluso se relaciona con las economías creativas, que con la industria cultural, se han apropiado del mismo.

\section{Conocimiento libre como cultura libre}

Al hablar de conocimiento libre se ponen bajo la lupa diversos aspectos del conocimiento humano, no como la capacidad de cognición ni como una forma en particular de generar saberes de manera estructurada, sino como el reconocimiento de que las culturas humanas tienen formas diversas de generar sus propias condiciones de vida, lo que incluye sus propios saberes, las formas de transmitirse, de incorporarse a los rituales comunes y también de transformarse en otros saberes. En este sentido, el conocimiento libre o compartido, es el reconocimiento de que hay aspectos de la vida humana que no pueden restringirse y tienen la capacidad de diseminarse libremente.

Sin embargo, autores como Stallman, o instituciones como EDRI (Derechos Digitales en Europa) o FCForum (Foro Internacional para la Cultura Libre), han reconocido las amenazas a la diseminación sin restricciones del conocimiento y los contenidos a partir de las condiciones legales y tecnológicas que las empresas que distribuyen y generan productos culturales, han creado, ya sea a partir de leyes que le prohíben a los usuarios realizar copias de archivos o con candados digitales (DRM). Es decir, las tecnologías que permiten una diseminación más alta de los contenidos, por su capacidad de reproducirse de forma rápida, al mismo tiempo han introducido mayores preocupaciones a las industrias culturales, lo cual al mismo tiempo inhibe otras formas de apropiación cultural que en épocas anteriores ocurría sin mayores problemas. 


\section{Conocimiento libre y autoridad}

Las formas de organización adhocrática y de inteligencia colectiva incentiva formas horizontales de organización en las que cualquier persona puede tomar decisiones, agregar y borrar información. Al hablar de autoridad, no se quiere recurrir necesariamente a la organización vertical o jerárquica en la que otro tipo de organizaciones definen sus contenidos a partir de la especialización de puestos, sino a que dentro de Wikipedia la autoría no está completamente definida, y los usuarios en sí no se consideran autores ni dueños de los contenidos, pues donan su trabajo.

En Wikipedia la autoría es secundaria e, incluso, queda oculta por la participación comunitaria, la inteligencia colectiva. Este es un rasgo que autores como Keen (2011) han criticado, pues consideran que menoscaba un sistema que funciona dentro de la academia y el mundo de las artes, en las que se sanciona las malas aportaciones y se reconoce sólo a las destacadas. Para quienes estiman el sistema de autor (el derecho a autorizar quién y cómo se usa una obra) Wikipedia pone en riesgo quién, cómo se utiliza y cómo se autoriza aquello que se considera valioso en la sociedad. Sin embargo, algo que desatiende Keen, pero sí le interesa a los teóricos del remix Ferguson, 2012; Gaylor, 2011 y Navas, 2012 y a los teóricos de la cultura libre, es que ni la autoría ni la figura del autor han existido siempre, sino que son invenciones europeas del siglo XVIII (Nimus, 2006; Shiner, 2004).

Asimismo, desde la perspectiva foucaultiana (Foucault, 1987), el estatuto de autoría se va modificando a lo largo del tiempo, pudiendo incluso recrear una genealogía de la autoría en la cual Wikipedia en español tiene una posición que, en principio, parecería opuesta a una posición tradicional, pero como se ha analizado, en la práctica no se puede oponer al sistema de autor (a través de las leyes del copyright), y como se ha analizado en el capítulo anterior sus reglas implican la verificabilidad, es decir, la información que ahí se construye debe de estar respaldada por el sistema de autoridad que se ha construido en la academia, la ciencia y las artes; el mismo sistema heredado de la modernidad ilustrada. Lo que Keen (2011) critica de este sistema, sin embargo, es que a pesar de que existan estas reglas, no hay un sistema editorial que vigile lo que se publica y que, por ende, haya errores, vandalismo y muchos estilos de escritura.

La autoría, como señala Foucault (1987), así como el sistema de derechos de autor, no sólo sirven para probar la relevancia y exactitud de la información, sino también como "un instrumento de dominación de las élites" (O’Neil, 2009: 2). El proceso de construcción de conocimiento colaborativo y abierto, tendría que ser diferente del proceso tradicional de la ciencia, o al menos poner en duda la neutralidad del conocimiento científico:

Ha sido ampliamente aceptado que la ciencia avanza mediante el uso de su propio método interno para conseguir la verdad, de modo que el conocimiento científico se legitima por su propia autoridad. Sin embargo, resulta que no existe tal método, y la ciencia se entiende mejor como una actividad de base cultural, es decir, como el producto de un proceso social. (Bortoft citado en Elbers, 2013: 68)

Desde la perspectiva de una política del disenso, es decir, una postura que apela al rechazo a una construcción del conocimiento por parte exclusiva de un 
sistema autoritario en el que hay un grupo que construye el saber y otro que sólo lo consume o está en subordinación, parecería entonces que Wikipedia se basa en un sistema disensual de participación.

Esto puede ser cierto parcialmente porque, como se ha mencionado anteriormente, el sistema de autoridad de Wikipedia, existe y es altamente especializado, debido a que los editores con experiencia están comprometidos con esa gobernanza interna y el sistema de reglas que se ha ido generando. En Wikipedia no se califican las credenciales académicas de los usuarios ni se pide que se identifiquen, sin embargo, hay una serie de factores que son evaluados en las ediciones de cada usuario para determinar si sus creaciones son o no son aptas. Este sistema no es ejecutado por un usuario en particular, sino por diversos usuarios que trabajan asincrónicamente.

La convención de Wikipedia en español resumida con WP: dueño o Propiedad de los artículos, señala que los contenidos creados por los editores no son propiedad de quien los comenzó ni quien aportó más ni de nadie en particular, y siempre serán susceptibles a ser modificados, corregidos y actualizados libremente por lector-editor que los considere conveniente. En entrevista, un voluntario de Wikimedia México comentó que en una de sus primeras ediciones, cuando aún no conocía las políticas del proyecto, firmó con su nombre el artículo que había editado, lo cual fue sancionado de manera poco amable por otro usuario experimentado. Esto ahuyentó por un tiempo al editor novato, sin embargo, más adelante continuó editando hasta absorber y replicar las reglas del proyecto.

Esta incapacidad de adjudicarse la autoría de los artículos de Wikipedia no es del todo cierta, pues en muchas ocasiones, los editores protegen celosamente los artículos que han creado, no permitiendo que otros usuarios modifiquen la redacción o alteren partes substanciales del mismo. Sin embargo, este tipo de comportamientos suelen ser temporales, pues la presión de la comunidad tenderá a respetar las políticas y convenciones de Wikipedia. Aunque la autoría no esté permitida abiertamente, los editores que crean artículos destacados (WP:AD) o artículos buenos (WP:AB), que son una categoría de artículos que han sido revisados y corregidos de tal manera que cumplen una serie de requerimientos extra, suelen colocar en sus páginas de discusión el número de artículos destacados que han creado, o el número de ediciones que llevan en el proyecto, lo cual también es otra forma de adquirir prestigio dentro del proyecto.

\section{¿Herramientas para debatir o para anular el debate?}

La deliberación es uno de los valores primordiales de Wikipedia, pues cada artículo posee una página de discusión (talk page en la versión inglesa) para que los usuarios viertan sus opiniones con respecto al contenido de los artículos. En algunos artículos, existe más información en las páginas de discusión que en el artículo mismo, pero en la mayoría de los casos, las páginas de discusión aún no son creadas o están vacías porque no ha habido ningún desacuerdo o debido a que se trata de contenido que no genera desacuerdos.

También los usuarios tienen páginas de discusión personales, donde otros usuarios pueden contactarlos, debatir algún tema, agradecer las contribuciones o notificar sobre asuntos de interés general. 
Existe, también, una herramienta llamada Café3 (Village pump en la versión inglesa) en el que los usuarios pueden debatir temas de interés general, proponer nuevas políticas o cambios de reglas, o tener amplias discusiones sobre temas delicados, como el bloqueo de usuarios, el comportamiento erróneo de algún usuario, etc., o sobre el contenido de algunos artículos.

Sin embargo, Wikipedia opera con varios sistemas de participación, como los son los procesos de votación para modificar las políticas de la misma enciclopedia. Aunque se trate de una enciclopedia abierta, a estos procesos de votación no puede acceder cualquier usuario ya que debe cubrir un mínimo de requisitos: tener al menos 500 ediciones, y llevar 6 meses dentro del proyecto. Estos requisitos mínimos no siempre han existido, pues en febrero de 2016 algunos usuarios prepararon una votación (la cual anteriormente se había discutido en el Café) para modificar la política anterior, que permitía que los usuarios con 50 ediciones y 4 días de antigüedad votaran. El cambió de política surgió debido a que notaron que había personas creando varias cuentas (cuentas títere) para alterar procesos de votación; sin embargo, no todos estaban de acuerdo con el cambio, pues consideraban que esto haría que menos usuarios quisieran participar en los procesos de votación.

Lo cierto, y de acuerdo a los datos obtenidos, los procesos de votación no atraen a muchos usuarios, pues la mayoría de ellos prefieren dedicar su tiempo en Wikipedia a editar contenido o a crear artículos nuevos. En promedio, de acuerdo a las 14 votaciones analizadas de 2004 a 2016, 72 usuarios participan en los procesos para modificar políticas o aspectos administrativos de Wikipedia en español; esto quiere decir que la participación es baja y no representa a los miles de usuarios que la editan, y mucho menos a los millones que la consultan (Tabla 2).

3 Véase: https://es.wikipedia.org/wiki/Wikipedia:Café/ 
Tabla 2. Número de usuarios que participaron en las votaciones analizadas de 2004 a 2016

\begin{tabular}{|l|l|l|}
\hline & Fecha de la votación & No. De usuarios \\
\hline 1 & 10 a 23 de febrero de 2016 & 91 \\
\hline 2 & 17 a 30 de octubre de 2015 & 108 \\
\hline 3 & 10 a 24 de diciembre de 2014 & 79 \\
\hline 4 & 7 a 22 de marzo de 2013 & 102 \\
\hline 5 & 6 a 21 de junio de 2012 & 89 \\
\hline 6 & 30 de enero a 13 de febrero de 2011 & 107 \\
\hline 7 & 15 al 28 de febrero de 2010 & 97 \\
\hline 8 & 2 a 17 de noviembre de 2009 & 91 \\
\hline 9 & 9 a 16 de mayo de 2008 & 77 \\
\hline 10 & 10 al 24 de octubre de 2007 & 69 \\
\hline 11 & 18 de octubre a 1 de noviembre de 2006 & 51 \\
\hline 12 & 5 a 15 de abril de 2005 & 12 \\
\hline \multirow{2}{*}{13} & 25 de noviembre a 11 de diciembre de 2004 & 22 \\
\hline \multirow{2}{*}{14} & 18 de agosto a 1 de septiembre de 2004 & 16 \\
\hline \multirow{2}{*}{} & TOTAL & 1011 \\
\cline { 2 - 2 } & $\%$ & $72.21 \%$ \\
\hline
\end{tabular}

Fuente: Elaboración propia

Por otro lado, lo que señalan los voluntarios de Wikimedia México quienes, además de editar, realizan en eventos para difundir la enciclopedia virtual, suelen participar poco en las votaciones, y de acuerdo al historial de ediciones de la mayoría de ellos, son sólo 3 voluntarios quienes participan regularmente en estos procesos. La mayor parte de los que no votan, señalan que no se trata de desidia o que consideren que no es importante, sino porque prefieren invertir el tiempo que le dedican a la enciclopedia en realizar otras actividades, especialmente editar, pero sobre todo participar en eventos, como editatones (maratones de edición de Wikipedia) o talleres.

Además de las votaciones, dentro de Wikipedia existen otras herramientas para vigilar que el contenido que se crea cumpla con los requisitos mínimos y con las políticas (especialmente la de relevancia enciclopédica y punto de vista neutral), como el historial de cada artículo, el historial de ediciones de los usuarios, y las páginas de cambios recientes y páginas nuevas. Estas páginas especiales muestran los cambios en tiempo real, lo que permite que los usuarios que realizan mantenimiento puedan monitorear para ver si alguien ha realizado vandalismo o ediciones no permitidas. Una vez que los usuarios están familiarizados con esta 
herramienta, saben que sus contribuciones pueden ser modificadas, lo que puede tener repercusiones a largo plazo.

Para algunos investigadores, este sistema es similar al esquema panóptico (Foucault, 1990) pues es un sistema de vigilancia en el que cualquier usuario puede observar y ser observado; sin embargo, hay usuarios que prácticamente se dedican al mantenimiento y a corregir los errores que consideran a partir de las políticas de Wikipedia (Ver Figura 1)

Figura 1. Esquema panóptico dentro de Wikipedia en español

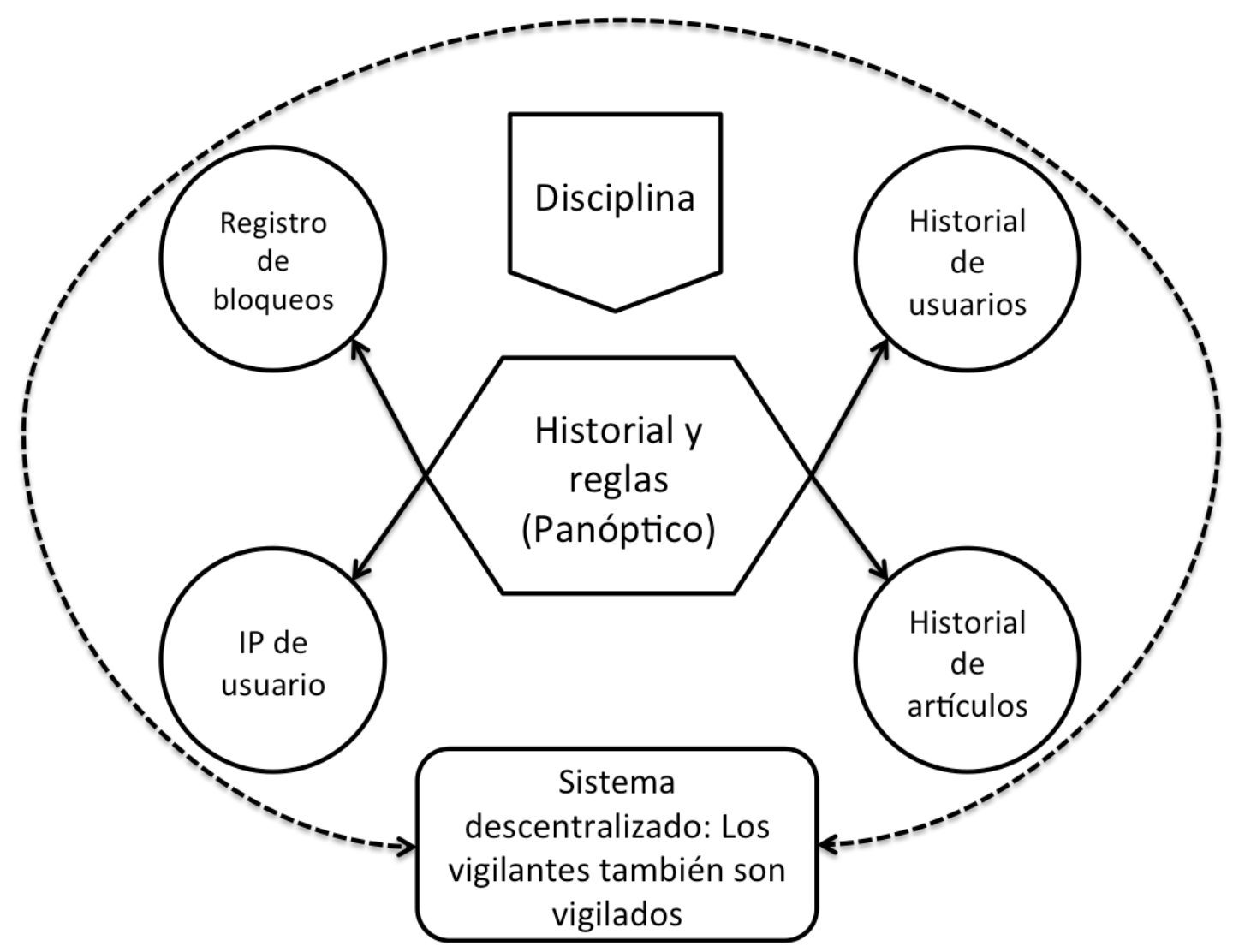

Fuente: Elaboración propia

En la práctica, el esquema de vigilancia puede ser muy efectivo para detectar errores y posibles sabotajes al proyecto; sin embargo, también lo utilizan otros usuarios para recordar viejas rencillas o errores del pasado que cometieron los usuarios.

Todo se ve y todo queda para el recuerdo, para tu detrimento; para que te dé vergüenza. (...) Luego es intimidante Wikimedia, de saber que tus contribuciones, tu nombre, tus datos viajan por todo el mundo. Son sitios tan visitados que, si no tienes como el cobijo... (Entrevista a wikipedista, 4 de julio de 2016). 
La apertura de Wikipedia, a pesar de que pudiera parecer contradictoria, trata de respetar el anonimato de las personas que participan. Lo que está bajo el escrutinio es la información, y aunque aparezca el nombre del usuario o su IP, no hay razones para que esa información se use para desacreditar a la persona que realizó la contribución, al menos en el discurso. Ha habido casos, sin embargo, donde los usuarios han tenido problemas por editar sobre temas sensibles, en especial cuando se trata de política.

\section{Los editores o gatekeepers}

Los editores de Wikipedia participan, no sólo por altruismo para mejorar la información dentro del sitio y así incrementar su credibilidad, sino también porque a diferencia de otros medios de comunicación o de generación tradicional de contenidos (libros, artículos científicos, etc.), la creación colectiva permite la posibilidad de tomar decisiones sobre lo que es o no es relevante dentro de estándares más abiertos. Si bien los pilares y políticas de Wikipedia parecen crear certezas sobre lo que puede o no publicarse, el último pilar ("Sé valiente" o "be bold") le otorgan a los editores una gama más amplia de opciones para elegir fuentes y modos de integrar sus perspectivas sobre un fenómeno.

En el caso de los temas de política y asuntos de interés público en Wikipedia en español, los usuarios son capaces de identificar la interferencia gubernamental o algún sesgo publicitario que pudiera restarle neutralidad a los artículos, borrando la información dañina, bloqueando a usuarios sospechosos y protegiendo artículos para que sólo sean editados por usuarios auto-confirmados (usuarios con mayor experiencia y credibilidad) de forma temporal. En esta investigación se identificaron ediciones de usuarios que modificaban y creaban artículos de políticos del estado de Sonora, los cuales fueron denunciados en el Tablón de Anuncios de los Bibliotecarios de ser cuentas bajo propósito que estaban vinculadas a un partido político (lo cual fue confirmado por usuarios que realizaron una investigación que vinculaba a ciertos usuarios con su actividad profesional fuera de Wikipedia), lo que infringe las políticas del proyecto. Dichos usuarios fueron bloqueados, algunos temporalmente y otros permanentemente.

Algunas de las decisiones administrativas que realizan los bibliotecarios, como los bloqueos a usuarios, si bien son medidas punitivas, son la última instancia, después de las discusiones y los intentos de llegar a otro tipo de acuerdos. Sin embargo, hay opositores a la forma en que las políticas de Wikipedia en español se llevan a cabo, señalando que el enciclopedismo y el punto de vista neutral son formas de estandarizar el conocimiento. "Algunos detractores rechazan el pilar del punto de vista neutral como un principio ideológicamente sospechoso, en particular dado el estricto cumplimiento de este que impone un extensivo aparato operativo." (Van Dijck, 2016; 233).

Sistema de recompensas dentro de Wikipedia

Aunque Wikipedia pueda ser vista como un actor en la sociedad red y, de esta forma, depende de otros actores que le dan visibilidad (Google con su motor de búsqueda) o de las compañías que conectan a los usuarios a Internet; también depende de los usuarios que visitan el sitio para leer y editar los contenidos, quienes a su vez poseen diversas motivaciones para editar. Sin embargo, lo que es 
posible aportar con este artículo, es que algunos de los editores más comprometidos con el proyecto consideran que deben velar por la calidad del contenido, neutralizando artículos, borrando datos maliciosos o perjudiciales, revirtiendo vandalismo; y aunque algunos quisieran que el proyecto se cerrase cada vez más para que no hubiera usuarios anónimos, el hecho de que existan los usuarios que hacen ediciones vandálicas es un incentivo más para que los usuarios participen.

Un ejemplo de estos incentivos es el concurso realizado por dos editores mexicanos, que también son voluntarios de Wikimedia México, quienes para incrementar la participación de los usuarios que revierten ediciones vandálicas, organizan un concurso de reversiones anualmente, el cual invita a los editores que hacen mantenimiento a que por un mes realicen la mayor cantidad de reversiones que puedan, y a los tres usuarios con más ediciones revertidas se les coloca un premio simbólico en forma de barnstar (estrella de granero).

Ortega Soto y Ramírez López ya han analizado los incentivos simbólicos dentro de Wikipedia en español, señalando que hay una correlación entre los editores más activos y el número de trofeos que acumulan así como el capital simbólico que poseen dentro del proyecto, sin embargo, lo que se ha corroborado en este análisis etnográfico es que los usuarios no sólo se motivan por el premio, sino por lograr una meta en común, realizar más reversiones que en la edición anterior, o simplemente luchar contra el vandalismo, que es visto dentro de Wikipedia en español como una amenaza constante. Como lo señala uno de los organizadores:

Dentro del ambiente, como esto es voluntario, para tener continuidad y mantener el interés, creo que es importante dar motivaciones, dar pequeños reconocimientos y pequeños regalos virtuales de manera simbólica de tal manera que te sientas participe de una comunidad y te sientas valorado por lo que haces. En este caso, en el concurso de reversiones fue involucrar a personas que normalmente están haciendo esta actividad, conjuntarlas y decir: si le dedicas bastante tiempo, registra cuánto estás llevando y las personas que estaban en primer lugar, se les da un pequeño reconocimiento virtual, una imagen o medalla en su página de discusión. De esta manera te estamos reconociendo el esfuerzo que estás haciendo para con el proyecto, que es enorme, que sabemos que es voluntario, que le estás dedicando parte de tu vida para que esto se mantenga y que lo agradecemos de esta manera. Creo que este tipo de concursos, no solamente de reversiones sino otros más, fomentan este tipo de cultura para mantener vivo el proyecto. (Entrevista a voluntari@ de Wikimedia México, 11 de febrero de 2017)

Por los comentarios de los usuarios, se puede apreciar que durante el concurso de reversiones, aumentaron las ediciones de mantenimiento, en especial las que buscan ediciones de vandalismo de usuarios anónimos.

\section{Activismo y conocimiento libre}

Algunos editores de Wikipedia en español y voluntarios de Wikimedia México consideran que su labor no sólo es mejorar el contenido del sitio, tanto en cantidad como en calidad, sino también se sienten parte de un movimiento de activismo 
social, que tiene el objetivo de aminorar la brecha de conocimiento al poner en circulación la información que sólo estaba disponible en medios impresos, pero también reducir las brechas sociales que se han creado dentro de Wikipedia y tienen relación con las fronteras simbólicas que se han creado.

Para los voluntarios de Wikimedia México, en especial a las voluntarias, la brecha de género es un asunto que tiene impacto en los contenidos de Wikipedia en español, pues al haber una menor cantidad de editoras, los contenidos son escritos a partir de una perspectiva masculina.

Debido a esto se han creado maratones de edición de Wikipedia exclusivos para mujeres a partir de 2015, denominados editatonas en los que se suelen editar biografías de mujeres, sobre feminismos u otros contenidos que podrían mejorar el contenido de la enciclopedia. En estos eventos se suelen enfrentar con algunos usuarios de Wikipedia, en especial porque al interpretar de manera cerrada algunas políticas, como la de relevancia enciclopédica y que el contenido debe estar basado en fuentes fiables y verificables, pues muchas veces el contenido sobre personajes femeninos no está disponible o es difícil de encontrar.

... cuando estás editando sobre mujeres (...) te enfrentas a que no hay referencia; qué hacemos, pues ya no se hace, te borran el artículo, te sientes mal, lo dejas. O sea es algo que también hemos diagnosticado las mujeres en Wikipedia que trabajamos este tea, que cuando el editor es hombre, generalmente no permite o insiste mucho en que borren un artículo, o sea que cada intento de borrado es como una batalla que tienen que ganar; y la mayoría de las mujeres lo dejamos: ya, editaré de otra cosa. (Entrevista a voluntari@ de Wikimedia México, 14 de septiembre de 2016)

Si bien, varios autores y autoras (Cohen, 2011; Collier y Bear, 2012; Forte, et al., 2012; Halfaker, et al., 2012; Pagola, 2013), y las propias investigaciones de la Fundación Wikimedia (Salvaggio, 2014) han analizado las razones y los perjuicios que existen en Wikipedia debido a la brecha de género, el trabajo de cada organización es particularmente diferente y se enfrenta a distintas barreras socioculturales. Las voluntarias de Wikimedia México han tenido que ir modificando su enfoque, debido a los eventos coyunturales a los que se enfrentan, como la violencia de género, el feminicidio y otros aspectos que consideran urgentes.

El 24 de abril de 2016, voluntarias y voluntarios de Wikimedia México salieron a las calles con dos propósitos, sumarse a la marcha llamada \#24A apoyando las consignas de las protestas, pero también para documentar el evento, y usar esas imágenes para ilustrar los artículos de Wikipedia y liberar imágenes con licencias libres en la plataforma Wikimedia Commons. Sin embargo, también llevaron consigo la consigna: "Invisibilizar a las mujeres de la historia también es violencia \#Editatona” (Ver Figura 2), con una clara alusión a la forma en que está redactada la Wikipedia en español y a las pocas biografías de mujeres comparadas a las biografías de personajes masculinos. 
Figura 2. Voluntarias y voluntarios de Wikimedia México en la marcha del 24 de abril contra las violencias machistas.

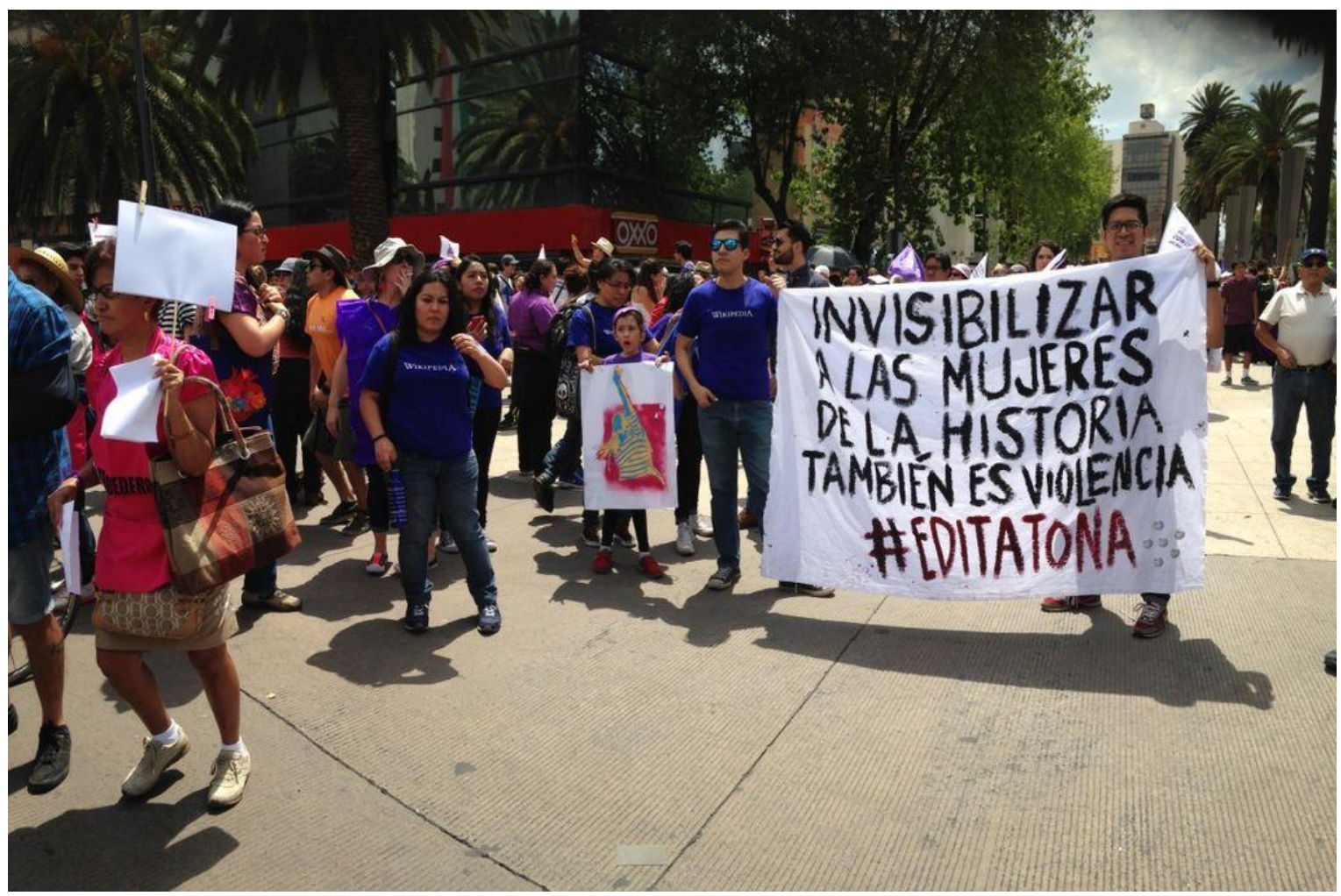

Fuente: Wotacito, CC by $4.0^{4}$

\section{Conclusión: El poder en red de los usuarios de Wikipedia}

Los usuarios de Wikipedia como parte del ecosistema de la información en Internet, poseen la capacidad de incidir en la construcción y difusión del conocimiento libre dentro y fuera de la red; sin embargo, como se ha señalado antes, existen diversas problemáticas alrededor. En primer lugar, los usuarios de Wikipedia en español provienen de lugares concretos, tienen ideologías para editar y también, como cualquier ser humano, pueden equivocarse; sin embargo, debido a que la herramienta es proclive al cambio continuo, cualquier tipo de información se puede modificar y mejorar, a pesar de que también exista la posibilidad de que parte de esos cambios sean erróneos.

Las políticas de Wikipedia, en este sentido, no son solamente reglas para cumplir, sino que también tienen un rol activo al ser especialmente maleables, porque deben interpretarse e, incluso, se pueden modificar si ponen el riesgo el futuro del proyecto. Aunque los pilares de Wikipedia no hayan cambiado desde que el proyecto surgió, el quinto pilar ha supuesto para algunos editores, la posibilidad de que la enciclopedia no sólo sea un almacén estéril de conocimiento, sino uno que represente las problemáticas y dinámicas de sociedades concretas.

4 Archivo disponible en:

https://commons.wikimedia.org/wiki/File:Editatoneras_en_marcha_24A.jpg 
Sin embargo, como se ha demostrado en la investigación, para que esto suceda se requiere que haya usuarios de una diversidad más amplia, tanto geográfica como ideológicamente, pues como cualquier otro sitio de creación de contenidos, entre más diversa sea su procedencia, los resultados serán más amplios. Por otro lado, existen voces dentro del proyecto que desean que la plataforma se cierre sólo a usuarios experimentados o a personas expertas en un campo específico, lo cual reduciría la apertura del mismo, contradiciendo los valores y factores que en primera instancia hicieron que creciera.

En conclusión, dentro de cada artículo de Wikipedia en español, el debate del contenido no está solamente en el producto informativo que se muestra en el espacio principal, sino en las discusiones, en el historial que muestra los cambios y en cada versión de la enciclopedia que crece a cada segundo para mostrar distintas caras de nuestra realidad.

\section{Bibliografía}

ADORNO, Theodor y Max Horkheimer (2007). Dialéctica de la ilustración. Akal.

ATTALI, Jacques (1995). Ruidos. Ensayo sobre la economía política de la música. Siglo XXI.

BOAL, Augusto (2013). Teatro del oprimido. Alba Editorial.

BOLLIER, David (2008). Viral spiral: how the commoners built a digital republic of their own. Nueva York: The New Press.

BRUNS, Axel (2008). Blogs, Wikipedia, Second Life, and beyond: From production to produsage. New York: Peter Lang.

CARPENTIER, Nico (2011). Media and participation: A site of ideologicaldemocratic struggle. Bristol, UK: Intellect Ltd.

CASTELLS, Manuel (2012). Comunicación y poder. México, DF: Siglo XXI.

CEFAÏ, Daniel, Marion Carrel, Julien Talpin, Nina Eliasoph, Paul Lichterman (2012). "Ethnographies de la participation". Participations. 3, 7-48.

COHEN, Noam (2011). "Define Gender Gap? Look Up Wikipedia's Contributor List". New York Times, January, 30(362), 1050-1056.

COLLIER, Benjamin y Julia Bear (2012). "Conflict, criticism, or confidence: an empirical examination of the gender gap in wikipedia contributions". En Proceedings of the ACM 2012 conference on computer supported cooperative work, ACM, 383-392.

DAHLGREN, Peter (2013). The political web: Media, participation and alternative democracy. Palgrave Macmillan Basingstoke.

DAWKINS, Richard (1994). El gen egoísta. Bogotá: Salvat.

DEBORD, Guy (2002). La sociedad del espectáculo. Valencia: Pre-textos.

ELBERS, Jörg (2013). Ciencia holística para el buen vivir: una introducción. Quito: Centro Ecuatoriano de Derecho Ambiental.

FERGUSON, Kirby (2012). "Embrace the Remix." at TED: Ideas worth Spreading. Video, 9:36. Recuperado de http://everythingisaremix.info/

FORTE, Andrea, Judd Antin, Shaowen Bardzell, Leigh Honeywell, John Riedl y Sarah Stierch (2012). "Some of all human knowledge: gender and participation in peer production." En Proceedings of the ACM 2012 conference on Computer Supported Cooperative Work Companion. ACM, 33-36.

FOUCAULT, Michel (1987). “QQué es un autor?”. Revista de la Universidad 
Nacional (1944-1992), 2(11), 4-19.

FOUCAULT, Michel (1990). Vigilar y castigar. Nacimiento de la prisión. Siglo XXI.

FREEDOM DEFINITION (2015). Recuperado el 2 de abril de 2017, http://freedomdefined.org/Definition

FRIEDMAN, Milton (2009). Capitalism and freedom. University of Chicago press.

GAYLOR, Brett (2011). RiP: A remix manifesto. (Documental). Canadá.

HALFAKER, Aaron, Stuart Greiger, Jonathan Morgan y John Riedl (2012). "The rise and decline of an open collaboration system: How Wikipedia's reaction to popularity is causing its decline." American Behavioral Scientis, 57(5): 664688.

HINE, Christine (2015). Ethnography for the internet: Embedded, embodied and every day. Bloomsbury Publishing.

HIPATIA. (s/f). "Segundo Manifiesto, reclama la libertad del conocimiento." $\begin{array}{llllll}\text { Recuperado el } & 1 & \text { de } & \text { abril } & \text { de }\end{array}$ https://hipatia.net/index.php?id=manifesto2_es

JEMIELNIAK, Dariusz (2014). Common Knowledge?: An Ethnography of Wikipedia. Stanford University Press.

JENKINS, Henry (2006). Fans, bloggers, and gamers: Exploring participatory culture. Nueva York: NYU Press.

JENKINS, Henry (2009). Fans, blogueros y videojuegos: la cultura de la colaboración. Vol. 180. Barcelona: Paidós.

JENKINS, Henry, Mizuko Ito, y Danah Boyd (2016). Participatory Culture in a Networked Era: A Conversation on Youth, Learning, Commerce, and Politics. Wiley. $\quad$ Recuperado https://books.google.com.mx/books?id=C_6aoAEACAAJ

KEEN, Andrew (2011). The Cult of the Amateur: How Blogs, MySpace, YouTube and the Rest of Today's User Generated Media are Killing Our Culture and Economy. Nicholas Brealey Publishing.

LESSIG, Lawrence (2005). Por una cultura libre: cómo los grandes grupos de comunicación utilizan la tecnología y la ley para clausurar la cultura y controlar la creatividad. Madrid: Traficantes de sueños.

LÉVY, Pierre (2004). Inteligencia colectiva. Por una antropología del ciberespacio, Washington DC: Biblioteca virtual em saúde.

LIH, Andrew (2009). The Wikipedia revolution: How a bunch of nobodies created the world's greatest encyclopedia. Hyperion.

MOUFFE, Chantal (2005). On the political. Psychology Press.

NAVAS, Eduardo (2012). Remix theory: The aesthetics of sampling. Springer Wien.

NIMUS, Anna (2006). Copyright, copyleft and the creative anti-commons. Berlín. Recuperado de http://subsol.c3.hu/subsol_2/contributorso/nimustext.html

O'NEIL, Mathieu (2009). Cyberchiefs. Autonomy and authority in online tribes. Pluto Press.

ORTEGA SOTO, José Felipe, y Joaquín Rodríguez López (2011). El potlatch digital: Wikipedia y el triunfo del procomún y el conocimiento compartido. España: Cátedra.

PAGOLA, Lila (2013). "De mujeres y enciclopedias. Formas de construir realidad y 
representaciones.” En Graciela Natansohn (Ed.), Internet en código menino. Buenos Aires: La Crujía.

POPPER, Karl (1992). La sociedad abierta y sus enemigos. Planeta-De Agostini.

RANCIÈRE, Jacques (2003). El maestro ignorante. Barcelona: Laerts.

RANCIÈRE, Jacques (2010). El espectador emancipado. Ediciones Manantial.

RANCIÈRE, Jacques (2015). Dissensus: On politics and aesthetics. Bloomsbury Publishing.

SALVAGGIO, Eryk (2014). Help us close Wikipedia's gender gap. Recuperado el 7 de mayo de 2016, de https://wikiedu.org/blog/2014/11/13/wikipedia-gendergap-education/

SHINER, Larry (2004). La invención del arte: una historia cultural. Barcelona: Paidós.

SMITH, Adam (1994). La riqueza de las naciones (1776). Madrid: Alianza.

STAKE, Robert (1998). Investigación con estudio de casos. Ediciones Morata.

VAN DIJCK, José (2016). La cultura de la conectividad: una historia crítica de las redes sociales. Buenos Aires: Siglo XXI.

WILLIAMS, Sam, y Richard Stallman (2010). Free as in freedom (2.o): Richard Stallman and the free software revolution. Free Software Foundation. GNU Press. 